\title{
Correction to: Tri-country translation, cultural adaptation, and validity confirmation of the Scored Patient-Generated Subjective Global Assessment
}

\author{
Nicole Erickson ${ }^{1} \cdot$ Lena J. Storck ${ }^{2}$ - Alexandra Kolm ${ }^{3} \cdot$ Kristina Norman $^{4,5} \cdot$ Theres Fey $^{1} \cdot$ Vanessa Schiffler $^{1}$. \\ Faith D. Ottery ${ }^{6,7} \cdot$ Harriët Jager-Wittenaar ${ }^{7,8}$
}

Published online: 1 September 2021

(c) Springer-Verlag GmbH Germany, part of Springer Nature 2021

\section{Correction to: Supportive Care in Cancer (2019) 27:3499-3507 https://doi.org/10.1007/s00520-019-4637-3}

The text body contained an error on page 3501 Paragraph 2. Sentence 2:

It read: "Native speakers $\geq 8$ years or higher not 8 years of age who had no previous experience with the PG-SGA before participating and who were willing and able to provide demographic information were asked to rate comprehensibility and difficulty of the first four boxes of the PG-SGA."

This should in fact read:

The original article can be found online at https://doi.org/10.1007/ s00520-019-4637-3.

Nicole Erickson

nicole_erickson@outlook.com

Lena J. Storck

Lena.Storck@ksw.ch

Alexandra Kolm

Alexandra.Kolm@fhstp.ac.at

Kristina Norman

kristina.norman@dife.de

Theres Fey

theres.fey@med.uni-muenchen.de

Vanessa Schiffler

vanessa.schiffler@web.de

Faith D. Ottery

faithotterymdphd@gmail.com

Harriët Jager-Wittenaar

ha.jager@pl.hanze.nl

1 Comprehensive Cancer Center, Ludwig-Maximilian

University Clinic, Munich, Germany
"Native speakers $\geq 18$ years of age who had no previous experience with the PG-SGA before participating and who were willing and able to provide demographic information were asked to rate comprehensibility and difficulty of the first four boxes of the PG-SGA."

Publisher's note Springer Nature remains neutral with regard to jurisdictional claims in published maps and institutional affiliations.
2 Departement Medizin, Kantonsspital Winterthur, Winterthur, Switzerland

3 Department Gesundheit, Fachhochschule St. Pölten GmbH, St. Pölten, Austria

4 Department of Nutrition and Gerontology, German Institute of Human Nutrition Potsdam-Rehbrücke, Potsdam-Rehbrücke, Nuthetal, Germany

5 Research Group On Geriatrics, Charite Universitätsmedizin Berlin, Berlin, Germany

6 LLC, Ottery \& Associates, Vernon Hills, IL, USA

7 Research Group Healthy Ageing, Allied Health Care and Nursing, Hanze University of Applied Sciences, Groningen, Netherlands

8 Department of Maxillofacial Surgery, University of Groningen, University Medical Center Groningen, Groningen, Netherlands 